\title{
Data driven improvements in public transport: the Dutch example
}

\author{
Niels van Oort ${ }^{1,3} \cdot$ Daniel Sparing ${ }^{1} \cdot$ \\ Ties Brands ${ }^{2,3} \cdot$ Rob M. P. Goverde ${ }^{1}$
}

Published online: 27 November 2015

(c) The Author(s) 2015. This article is published with open access at Springerlink.com

\begin{abstract}
Due to reduced budgets, higher political expectations and increasing competition between operators, there is growing pressure on public transport companies and authorities to improve their operational efficiency. It is thus of utter importance for them to be able to identify inefficiencies, bottlenecks and potentials in their public transport service. Recorded operational data, which has quickly become more widespread in the last decade, aids greatly in this process and enables operators and authorities to continually improve. In this paper we identify some of the arising possibilities. We first describe the state of publicly available transit data, with an emphasis on the Dutch situation. Next, the value of insights from Automatic Vehicle Location data is demonstrated by examples. Thereafter, a software tool is presented that enables operators and authorities to quickly perform comprehensive operational analyses, and which was able to identify several bottlenecks when applied in practice.
\end{abstract}

Keywords Public transport $\cdot$ AVL data $\cdot$ Service reliability $\cdot$ Monitoring

\section{Introduction}

Similar to other countries, public transport in the Netherlands faces substantial costcuttings. Tighter overall budgets mean funding is being reduced, which makes it even more challenging to improve the quality and capacity, as required by the

Niels van Oort

NvOort@Goudappel.nl; N.vanOort@tudelft.nl

1 Delft University of Technology, Stevinweg 1, 2628 CN Delft, The Netherlands

2 University of Twente, Enschede, The Netherlands

3 Goudappel Coffeng, Mobility Consultants, Deventer, The Netherlands 
demanding (potential) customers. With the right data and tools it is not impossible, though. The key factors to enhanced and more cost-efficient public transport are travel time and service reliability. More insights into the mechanisms of these aspects are available by analysing several new data sources. Transport planners are starting to consider how data retrieved from automatic passenger counting systems (APC, such as passenger smart cards, see e.g. Neema et al. 2015; Pelletier et al. 2011) automatic vehicle location systems (AVL, see e.g. Furth et al. 2006; Hickman 2004) and mobile phones (Calabrese 2011) could improve the design of public transport networks and timetables and improve operations by predicting ridership in different scenarios (see e.g. Van Oort et al. 2015b). In addition, social media data, such as user data of Facebook, Twitter and Flickr, may yield new knowledge on public transport usage (Bregman 2012). Video, Bluetooth and Wi-Fi trackers may provide new insights into pedestrian flows in stations, at platforms and in vehicles (van den Heuvel et al. 2015).

The objective of this paper is to provide an overview of unreliability causes and to illustrate the potential benefits of data, focusing on Dutch AVL-data, obtained by a nationwide system. The novelty of our approach is the availability of one approach for the whole country (all Dutch AVL data handled in one way), next to the ability to automatically find bottlenecks and potential benefits. The first step in our approach was to investigate service reliability and its causes and impacts on passengers. The next step was to find and process data that clarifies and quantifies these. We developed a tool to translate the data into valuable information about quality of service. Finally, we applied the data and the tool to support several improvement plans of actual public transport services.

This paper presents examples from the Netherlands, where new data sources became available recently. The challenge is to transform that data into information that can be used for service improvements and thus to make optimal use of the value it possesses. For instance to identify concrete examples of how the network could be improved or the schedule optimized.

The outline of this paper is as follows: First, service reliability impacts on passengers are presented, including causes and improvement measures. Section 3 describes the available data in the Netherlands and the next section shows the opportunities to analyse that data. Section 5 presents a tool that supports processing the data and Sect. 6 shows the opportunities if APC data is added to the analysis. The final section presents the main conclusions, followed by acknowledgements and references.

\section{Service reliability}

\subsection{Passenger impacts}

Service reliability, which is a result of variability in operations (Abkowitz and Engelstein 1983; Ceder 2007; Mazloumi et al. 2010), is the certainty of service aspects (such as travel time) compared to the schedule as perceived by the user (Furth and Muller 2009; Van Oort et al. 2012). Unreliability causes longer and 
uncertain passenger journeys (Bates et al. 2001; Noland and Small 1995; Van Oort et al. 2012). The level of service reliability affects several choices made by travellers, such as mode and route (Schmöcker and Bell 2002; Turnquist and Bowman 1980). In numerous studies, reliability-related attributes have been found among the most important service attributes in a variety of situations. Balcombe et al. (2004) even report that passengers consider service reliability twice as important as frequency. König and Axhausen (2002) conclude that research over the last decade shows that the reliability of the transportation system is a decisive factor in the choice behaviour of people.

Even though reliability is thus considered of key importance, in Van Oort et al. (2012) it is demonstrated that passengers presently experience an insufficient level of service in this field. It is revealed that service reliability impacts on passengers depend on three main aspects, as shown by Fig. 1:

- How does the vehicle operate (compared to the schedule)?

- How do passengers travel through the network?

- How do passengers behave (concerning arrival at the first stop and transferring)?

Much scientific research and public discussion is being conducted concerning the impact of reduced societal costs for passengers of enhanced reliability in cost-benefit analyses (Van Oort et al. 2015a). These costs consist of passenger appreciation of a unit of time (variation) and the quantity of this time (variation). Most research (e.g. Li et al. 2010) focuses on the first part, while the latter, for instance reduction in (standard deviation of) travel time in a network context, still lacks insights (OECD/ International transport forum 2010; Van Oort et al. 2015a). Contrary to car traffic (Snelder and Tavasszy 2010), calculating the quantity aspects for public transport is complex, since a schedule, waiting and transferring are involved. New data sources, as described in this paper, are helpful to calculate these aspects.

\subsection{Causes and impacts of service unreliability}

\subsubsection{Causes}

In order to improve public transport in general and its service reliability in particular, the main causes of disturbances must be understood. The next sections

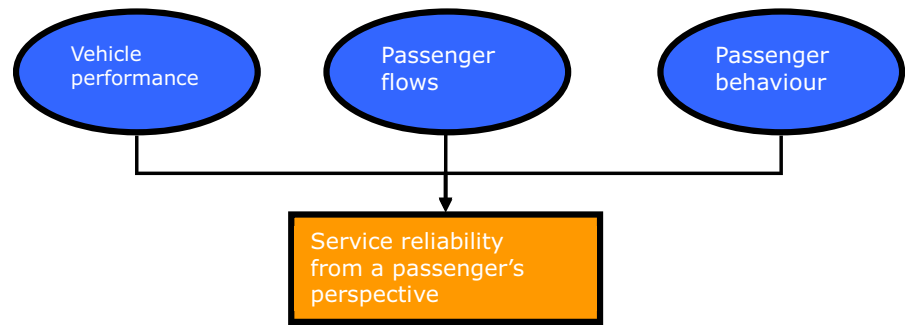

Fig. 1 Aspects affecting service reliability from a passenger perspective 
identify and analyse the main reasons for unwanted variation in public transport services and divides them into three components: namely driving time, stopping time and dwell time. It also distinguishes between the causes that are external and those that are internal, in order to clarify which actor is able to improve which element. This is illustrated by Fig. 2. These causes and relations are found by literature study (references will be provided in the next sections), by research and experiences of ourselves (Van Oort 2011) and by interviewing several authorities and operators via an international survey (Van Oort 2011, 2014). The fundament of our own research was data analysis (AVL and APC) of bus and tram lines in The Hague. In addition, field observations and interviews with drivers, planners and designers were performed enabling to derive the relationships.

Shalaby et al. (2001) found that trip time variation not only depends on service trip time (or line length) itself, but is also affected by the number of stops made, the number of signalized intersections passed, the vehicle intensity and the capacity per lane. Abkowitz and Engelstein (1983) found that line length, passenger activity and the number of signalized intersections affect average trip time. Most researchers agree on these basic factors (Abkowitz and Engelstein 1983; Levinson 1983; Abkowitz and Tozzi 1987; Strathman et al. 2000).

Considering the trip time components individually, we observed and analysed the different processes during a vehicle trip (of bus and tram in The Hague) to gain insights into the causes of trip time variability (Van Oort 2011), in addition to performing an international survey (Van Oort 2011, 2014) and studying literature. The first process we analysed was the driving between stops. This includes accelerating, braking and unplanned stopping. Since the causes of stopping may also be responsible for slowing down vehicles, both components are presented in one

Internal causes

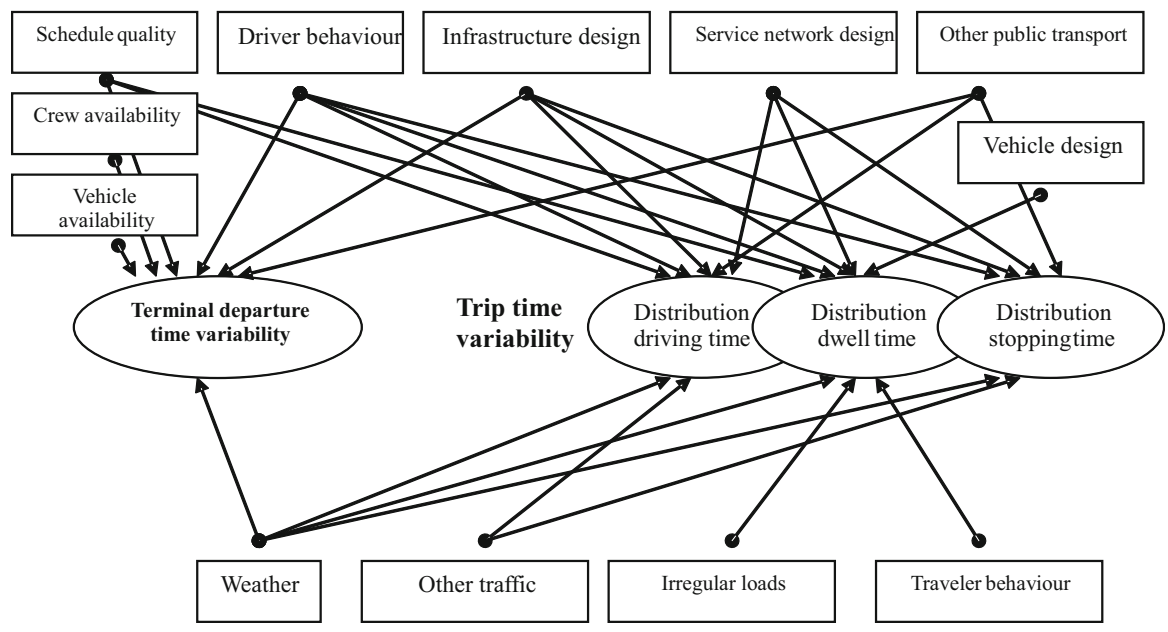

External causes

Fig. 2 Main causes of service variability in urban public transport 
overview. The causes presented below are responsible for actual driving time and stopping time variability.

\subsubsection{Driving and stopping time}

Concerning driving and stopping times, we found the following main internal causes:

Driver behaviour. The basic driving style of every driver differs. Some tend to be more passive, whereas others are more aggressive. This results in variances in speed, stopping and braking of vehicles traveling along the transport network and thus in faster or slower journeys for passengers.

Other public transport. Both on the same route as on intersections, other public transport routes may affect the driving and stopping time variability. The influence is usually at its largest at signalized sections. Especially when frequencies are close to the theoretical capacity of a track, lane or intersection, this increases the probability of delays and increased service variability (Goverde et al. 2001; Van Oort and van Nes 2010; Landex and Kaas 2009).

Infrastructure configuration. The infrastructure (stops, lanes, intersections, terminals) may be designed in such a way that service variability could occur, for instance when the capacity of infrastructure is not sufficient to accommodate all traffic, or as a result of interaction with public transport at intersections. This could result in delays for (some) vehicles and therefore in increasing variability (Kanacilo and van Oort 2008; Van Oort and van Nes 2010).

Service network configuration. The configuration of the service network may influence the service variability. Examples are the number of lines on the same route, their length and the number of stops (Van Oort and van Nes 2009). The type of service network configuration may enforce other causes, for instance the impact of other traffic and driver behaviour. Longer lines, for instance, affect all causes mentioned. In the schedule, multiple lines may be presented as a higher frequent, coordinated service, while in practice service variability increases due to interaction between the different routes. Another example is the synchronisation of lines and schedules, which may be introduced to ensure transfers. This dependency of lines may lead to additional variability, since delays are transferred between lines.

Schedule quality. The schedule may affect the way drivers operate. If the quality of the schedule for instance is insufficient and margins are too small, some drivers will speed up to stick to the timetable, whereas others will operate like they are used to. This behaviour can introduce service variability (Van Oort et al. 2012).

The main external causes with regard to driving and stopping times are explained below:

Other traffic. The interaction with other traffic causes service variability as nearly every vehicle, at least to some extent, faces different operational conditions. This mainly occurs at intersections (regardless if they are controlled with traffic lights or have to cope without) or where public transport services share tracks or space with other transport modes or pedestrians. The extent to which this cause affects driving and stopping time variability depends on the level of dedicated 
public transport infrastructure. This may vary from at grade to exclusive lanes (with shared intersections) or mixed operations.

Weather conditions. Different kinds of weather and the resulting changes in driver behaviour may result in variability (Hofmann and Mahony 2005). This mainly occurs when the weather is not in the regular state since this disturbs regular processes.

\subsubsection{Dwell time}

The following internal causes for variability in dwell times may be distinguished (if not explicitly mentioned above):

Driver behaviour. The driver behaviour concerning opening and closing doors and the extent to which the vehicle waits for late arriving passengers is of influence on service variability.

Vehicle design. Weidmann (1995) and Fernandez (2010) showed the impact of the number and positioning of doors in vehicles, to obtain an optimized dwell process. Vehicles and/or platforms enabling same level boarding and alighting are also of influence and the same goes for the method of ticket handling. A suboptimal design, related to passenger behaviour, may result in dwell time variability.

Platform design. The platform design is also important because it affects passenger behaviour. The design may lead to a better distribution of passengers along the platform, enabling an optimal dwell process. Width, length, location of sheds and other facilities are important elements and if design is suboptimal, variability may arise.

The main external causes we found, are:

Passenger behaviour. Different types of passengers have different boarding speeds (for instance due to age, experience, luggage). The way passengers make optimal use of a vehicle's doors is important, too. This is related to vehicle and platform design.

(Irregular) loads. Due to a different number of people boarding and alighting for every single trip, variability of dwell times will occur. Also crowding is an important cause of unreliable dwell times, being a result of large loads, passenger behaviour and vehicle and platform design.

On an aggregated line level, the number of stops is of importance, too. More stops usually cause a higher level of service unreliability. Thus line length is important, as is stop spacing.

The causes mentioned above are of importance when improving the level of service reliability as they identify the areas where enhancements may be applied. Quantifying the impacts of these causes is the next step. The following section deals with available data and improvement measures.

\subsection{Improving service reliability}

Lots of research has been carried out to identify the potential of improving travel time and service reliability. One could, for instance, think of improvements of vehicles, infrastructure, planning and operations. Literature shows that in urban 
public transport, substantial attention is given to ways to improve services at an operational level (Vuchic 2005; Ceder 2007; Cats 2014). Concerning strategic and tactical instruments, much research is already available. A lot of this focuses on the implementation of bus lane schemes, synchronisation and traffic signal priority, which are the most common solutions, as shown by e.g. Waterson et al. (2003). Ceder (2007), Vuchic (2005), and Lee et al. (2014) all present the different methods and effects and also give an overview of the issues which need to be considered in synchronisation. Potential instruments for improving operational quality during the design of the schedule are optimising trip time determination and holding (Delgado et al. 2012; Xuan et al. 2011).

Less researched so far, but also important, are the potential instruments that are available during network design, for instance, line length and design of terminals (Van Oort and van Nes 2009, 2010). Our study of a new tram line in Utrecht, the Netherlands (Van Oort 2012), showed that about $65 \%$ of the (societal) benefits are related to service reliability aspects, coming down to a sum of over $€ 200$ million during the total lifetime of the tram infrastructure. On busy bus trunks in Utrecht, a reduction of $30 \mathrm{~s}$ of trip time per bus saves about $€ 100,000$ to $€ 400,000$ in operational costs per year (based on costs per hour of operation of $€ 100$ ).

The first step to increase operational performance is a proper analysis of historical operations. This paper focuses on bus and tram operations. The performance for heavy railways based on track occupation data is described in (Goverde and Meng 2011). AVL systems (Strathman et al. 2000, Hickman 2004) are of great help as these provide databases of historical performance with regard to travel time and reliability. Although such data have already been available for years, it is only recently that these can be accessed and used by Dutch transit authorities, researchers and developers. In addition to facilitating analysis of performance, these data also enable forecasts of future service quality (Kanacilo and van Oort 2008; Wilson et al. 2009). In this paper we present practical examples of data to illustrate the usefulness of these kinds of analyses: several bottlenecks are identified, providing transport authorities with insight into determining investment priorities. Next step is to add data from smart cards (APC; Pelletier et al. 2011) to gain insights into passenger impacts.

\section{Public transport data}

\subsection{Timetable data}

Public transport companies have always dealt with large amounts of data when designing timetables, scheduling vehicles and staff, collecting fares and more recently tracking vehicle locations. However, it has only recently become possible to store large amounts of historic vehicle location and fare collection data, and therewith to utilize these data for analyses. Furthermore, in line with other "Open Data" initiatives in the public sector, data related to public transport is being made publicly available in more and more areas, notably in North America and more recently in certain European cities. 
The first type of public transport data that became publicly available is timetable information. Besides supplying public transport route planners with timetable data, computer-readable timetable information also allows for efficient analysis and comparison of public transport networks, describing, for instance, spatial coverage, commercial speeds, frequencies, and connections to adjacent public transport networks. However, timetable information does not yet provide insights into the performance of the actual operation and hence the service reliability of public transport and the real-time timetable information.

Accurate real-time vehicle location data (AVL) has become available for public transport operators with the wide availability of GPS- and mobile phone devices. AVL-data has also become publicly available in many areas in recent years, albeit often with the condition that it can only be used for passenger information. Early examples include the transit agencies of Washington, Boston and various US bus companies. We note that these days most Western public transport operators provide some kind of real-time vehicle location (or expected vehicle arrival time) to the public, but often this information is not yet technically or legally available for storing or further processing by third parties.

\subsection{The Dutch example: GOVI data}

In the Netherlands, most public transport operators take part in the Borderless Public Transport Information initiative [Grenzeloze Openbaar Vervoer Informatie in Dutch, GOVI (http://govi.nu)], aimed at making a wide range of public transport information publicly available in the Netherlands, ranging from timetables to fares, vehicle location and punctuality. The data exchange interfaces are defined by the set of standards called BISON (http://bison.connekt.nl/), which are the same for all participating public transport operators, including buses, trams, metros and regional trains. These standards are connected through the BISON architecture. Each of the standards describes an aspect of public transport operations, like the timetable, punctuality, actual departure and arrival times, dynamic platform allocation or free text. Another source of open public transport information, such as a GTFS (i.e. General Transit Feed Specification) feed on the national level, is the company 9292 REISinformatiegroep BV (http://9292opendata.org/), a company owned by Dutch operators that specializes in providing passenger information. The formats of GTFS and BISON are not the same, but the data included in both formats is mostly similar. Since both formats are well defined, scripts to automatically convert data from one format to the other exist.

GOVI was designed to facilitate data communication between vehicles and the land side to enable the provision of dynamic passenger information (i.e. real-time information). As an additional benefit, the actual and scheduled vehicle positions and times are logged in a database. Typically, for one transit line this involves 3000-7000 arrival times and departure times per day. Although this database was not the main objective of GOVI, it is a helpful source when monitoring and analysing public transport performance. The arrival times and departure times of vehicles at stops are recorded with a precision of 1 s. 3-8 \% of these time points are missing. This includes errors and outliers, since these are detected and denoted as 
missing by the GOVI organisation. Reason for missing data may be a malfunctioning GPS device or the wrong coupling of a vehicle number and a run number.

In 2012, the first Dutch public transport operator agreed to legally release AVL data via GOVI for storage and analysis by third parties, such as researchers and developers (http://webwereld.nl/cloud/56325-gvb-maakt-real-time-reisinformatieopen-data). Since then several other operators joined. The resulting data streams are publicly available via the Dutch OpenGeo Foundation (http://www.openov.nl/). The source of the data presented in this paper is the transit authority or via OpenGeo.

\section{Insights from Dutch AVL data}

\subsection{Data structure}

Automatic vehicle location data provides valuable insights into the level of service aspects such as travel time and service reliability. As a first step, it is important to understand the structure and the quality of the data source. In our case, AVL data (i.e. GOVI data) was available for several months from multiple operators in the format described by interface KV6 of the BISON standard mentioned earlier. An example extract of the most important data attributes and the first and last few records of a single public transport vehicle trip is presented in Table 1. We preferred GOVI data over other sources due to its availability and consistency.

This data table consists of time-stamped messages of important events on a trip. In particular, a trip starts with an INIT initial message and ends with an END message and all departures are logged with a DEPARTURE message. For some stops an ARRIVAL message is recorded, too, allowing for an estimate of the dwell time. Furthermore, in case there is no departure and arrival event taking place for a longer time duration (about a minute), an ONSTOP or an ONROUTE message is logged, including the exact location. Our data source already includes a value for delay, which comes down to the difference of the message timestamp and the planned arrival or departure time.

Table 1 Example data output from BISON interface KV6

\begin{tabular}{lllllll}
\hline Time & Message type & Operator & Line & Journey & Stop & Punctuality \\
\hline $08: 29: 00$ & INIT & $\ldots$ & B120 & 7001 & 99990140 & \\
$08: 29: 00$ & ONSTOP & $\ldots$ & B120 & 7001 & 99990140 & 60 \\
$08: 29: 22$ & DEPARTURE & $\ldots$ & B120 & 7001 & 99990140 & 82 \\
$08: 31: 28$ & DEPARTURE & $\ldots$ & B120 & 7001 & 99990290 & 88 \\
$\ldots$ & & & & & & \\
$08: 51: 04$ & ONROUTE & $\ldots$ & B120 & 7001 & & -202 \\
$08: 52: 37$ & ARRIVAL & $\ldots$ & B120 & 7001 & 99990500 & \\
$08: 52: 37$ & END & $\ldots$ & B120 & 7001 & 99990500 & \\
\hline
\end{tabular}




\subsection{Line-based analysis}

A commonly used visualization (Furth et al. 2006; Van Oort and van Nes 2009) of the performance of a transit line is plotting each trip as a line chart in a coordinate system of stops versus delay. Figure 3 (left) shows 1 month of bus trips of a certain line, as well as the median and 15th and 85th percentile. Such a chart reveals both the level of variations in the operation and the quality of the timetable and the systematic deviations. In detail, this chart visualizes that there is a good general ontime performance on this line, with occasional large delays but only very few buses substantially ahead of schedule. One can also observe that there are quite a few holding stops where even early buses wait for their scheduled departure time. Nevertheless, the delay slightly accumulates on average in the second part of the line. Another phenomenon that is shown by this chart is the ample time reserve used just before the last stop in order to be on time when punctuality is measured.

Another way to look at the same data is to plot vehicle headways instead of delays. A high frequency line with consistent delays but regular headways remains attractive to passengers. The chart is shown in Fig. 3 (right), the scheduled headway is $10 \mathrm{~min}$. This location-headway chart points out the regularity of high-frequency services along the line, as well as possible bus bunching. In detail, this chart visualizes that the headway deviations stay constant in the first two-thirds of the line, but the headway variance increases in the last third. Together with the previous chart, this indicates that interventions for punctuality and regularity are most useful towards the last part of the line.
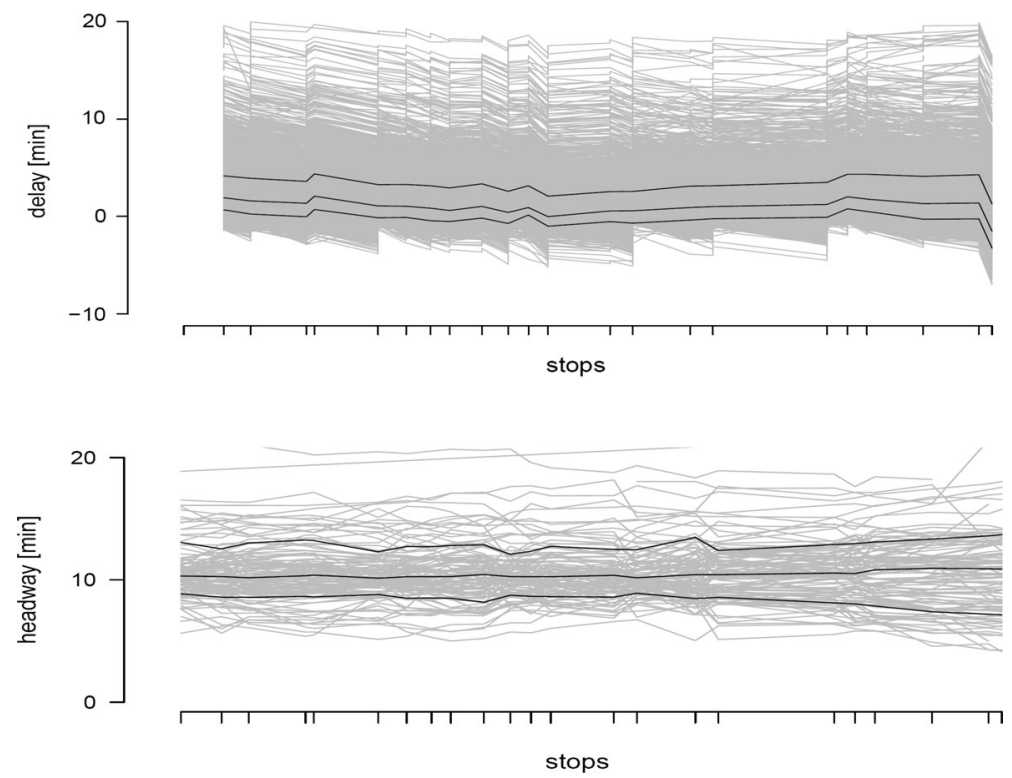

Fig. 3 Vehicle delays (upper) and headways (lower) along a single route 


\subsection{Network-wide analysis}

The ubiquitous availability of vehicle locator devices allows one to take a step further from line-based performance evaluation and investigate patterns on network level. Phenomena only visible on this level are the reliability of transfers, arearelated issues and possible bunching or interference on shared sections. An example of a network-level data visualization is the average delay at each stop, including stops with several transit lines, as shown on Fig. 4 (delay averaged over all days of January 2013).

In detail, the implication of this chart is that while there is a good general punctuality along the network, there are a few problem areas where a whole series of stops suffer from delays from all related bus lines, for example in the bottom left corner of the chart with lots of orange. On the other hand, there are a few isolated green points where buses tend to be often early - this problem could be solved once known by a simple adjustment in the published departure time.

\subsection{Inter-operator transfers}

An aspect of public transport travel that previously was invisible to the public and to operators, albeit of substantial importance to the passenger, is the reliability of transfers between operators or modes, such as between a long-distance train and a local bus. With open data, it is possible for anyone (so also for any operator) to investigate the actual reliability of inter-operator transfers and to take steps if necessary.

Figure 5 shows a discrepancy between vehicle punctuality and passenger experience, for a transfer that is scheduled to take 5 min excluding walking time [at station Hilversum Sportpark where there is one bus line and two train lines (all operated in a 30 min headway)]. The chart shows average values across all Sundays in January 2013, between 10 a.m. and 11 p.m. It is common that a public transport timetable includes a substantial time reserve before an important stop, and therefore as the left part of the figure shows, vehicles are consistently early at the transfer stop. However, this means that the passengers structurally have to wait much longer at the transfer stop than they expect from the timetable. As waiting time on the platform is perceived as much less comfortable as in-vehicle time (Van Der Waard

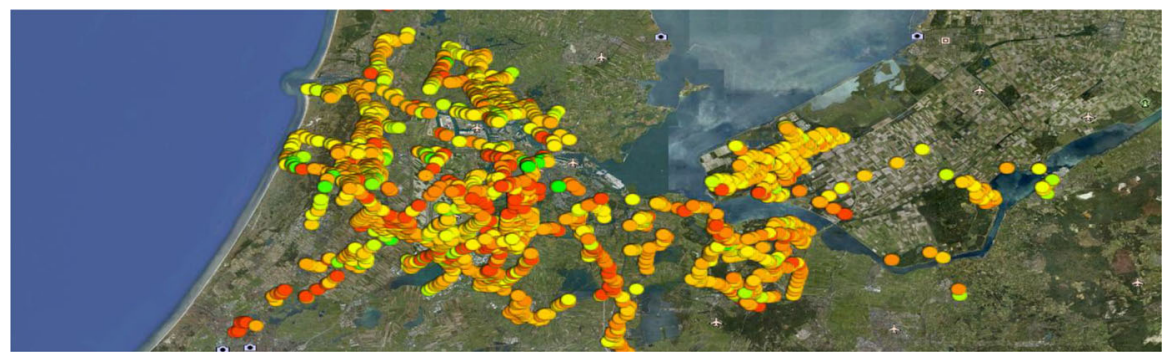

Fig. 4 Average delay per stop (green early, yellow on time, red late) (colour figure online) 

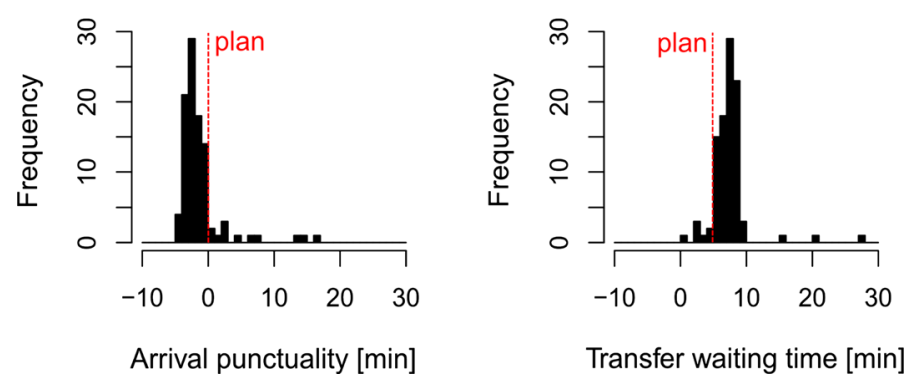

Fig. 5 Arrival punctuality of a vehicle and transfer waiting time for the passenger at a transfer location

1988), this implies that trips including this transfer are perceived of as being of less quality than expected from the timetable.

The relevance of open AVL data with regard to improving transfers is the following: open information on the reliability of inter-operator transfers enables any operator and transport authority to gain insight into the reliability of these transfers and take steps if improvement is required, such as synchronising timetables, holding vehicles in case of minor delays and informing passengers. See Sparing and Goverde (2013) for example for identifying transfers of interest and choosing which vehicles to hold in a multi-operator setting.

\subsection{Enriched travel advice}

As we covered in our introduction to open transit data, the original reason for opening up actual departure and arrival time information was to provide real-time passenger information. Collecting historical data, on the other hand, used to be against most terms and conditions of data providers and these are often considered sensitive business data. With the new developments, however, that also allows access to data for historical analyses, it is possible to calculate distributions of running times, arrival times and transfer waiting times, as demonstrated in the previous paragraphs. Building on these distributions, and returning to passenger information, it is possible to enrich travel advices to provide estimations on the reliability of public transport trips as follows.

Let us assume that probability distributions of departure and arrival times are available for all transit lines at all stops at all times. For example, fitting a normal distribution on all arrivals and departures in the past 3-6 months on the given type of day (working day/Saturday/Sunday) at the given time period (morning peak/ midday/afternoon peak/evening) for the given line, stop and direction can be a good first estimate. Furthermore, we assume for simplicity that the times of different lines are independent from each other.

Then for a travel advice with no transfers, we can provide the passengers with the distribution of the departure and arrival times. In fact, considering what really matters to the passenger, we can summarize these two distributions using two indicators: (1) the probability the vehicle departs too early and (2) the probability the vehicle arrives on time at the destination stop (within a certain time tolerance). If 
necessary, we can further simplify these indicators to a "traffic light" approach: colour coding the favourable, medium and low-reliability options.

In case of a travel advice including transfers, we can add an indicator for each transfer: the probability that the transfer is possible. Note that from the passenger's perspective, independent arrival and departure time distributions are irrelevant during a transfer, except for whether the transfer is possible or not.

See Fig. 6 for an example for such an enriched travel advice.

\section{The GOVI-tool}

To generate helpful insights from AVL-data, we developed a tool that translates the data into information and knowledge: the GOVI-tool. The GOVI-database consists of all actual and scheduled arrival and departure times at all stops of all trips of the participating public transport operators in the Netherlands. This implies large data sets: a month contains 100,000-200,000 records per line. Per stop and per trip, additional data are calculated based on the actual and scheduled arrival and departure time. Firstly, the actual dwell times per stop are calculated by subtracting the actual departure and actual arrival time. Secondly, the punctuality (both arriving at stops and departing from stops) is calculated by the difference between the actual and scheduled times. Thirdly, travel times on sections between two stops are calculated. Because the distance between each stop pair is included as well, the speed per section is calculated. The tool can cope with missing data, because in most GOVI-data a few percent of the records is empty. The number of observations per time period is reported, so that the analyst is able to judge whether the data produces results that are statistically reliable enough. Due to several operations within one line (i.e. operating with double frequency on a part of the line) these numbers may vary strongly.

To gain insights into the performance, mean values, and 15 th and 85 th percentile values are calculated for dwell times, for travel times on sections between stops and for punctuality at stops. This way, next to information on the average, information

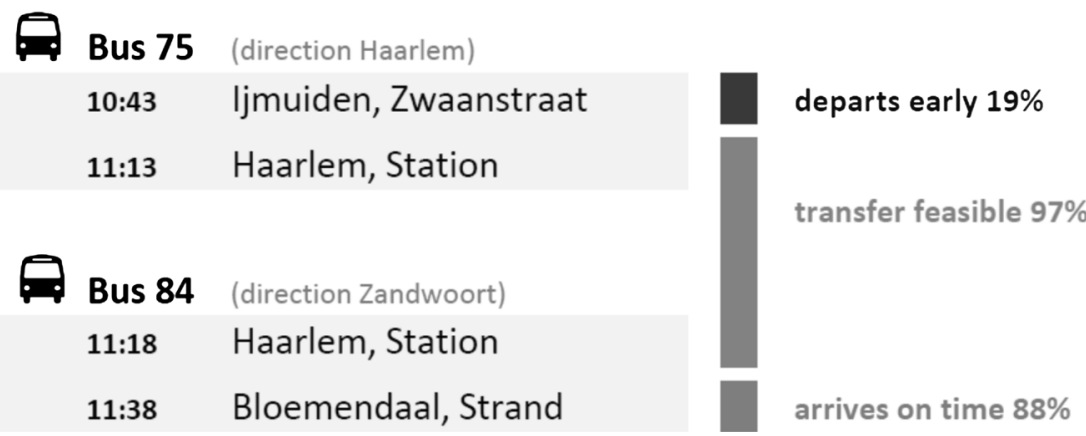

Fig. 6 Enriched travel advice including probabilities of early departure, transfer feasibility and on time arrival 
about variability is provided. The tool also provides information on cumulative values, such as total trip time, thereby illustrating the quality of actual performance compared to the schedule along the line. All information can be presented per stop, line and per direction, period of the week (working day, Saturday or Sunday) and period of the day (AM peak, PM peak, daytime or evening).

In addition to presenting the data in tables and figures, the tool is also capable of identifying bottlenecks. The tool easily finds its way through all the data and selects (predefined) outlier values. It could, for instance, present a list of all stops where the average dwell time is more than $30 \mathrm{~s}$ or where the schedule deviation is below zero (i.e. vehicles operating ahead of time). Bottlenecks with regard to service reliability may be found by selecting all stops where the difference between 15th and 85th percentile values of the punctuality deviation is higher than a chosen value.

The aforementioned GOVI-tool has been used in the Utrecht region to analyse all bus lines, gaining insights into the actual performance and the largest bottlenecks. Insights with regard to driving, dwelling and punctuality were generated for four periods per working day and for Saturday and Sunday (March 2012). Several performance indicators were compared to the Sunday values to gain insights in the maximum improvement potential. Below, we present examples of the generated graphs from both Utrecht and another line in North Holland (Figs. 7, 8, 9): punctuality along a line, travel speed between stops and dwell times. These figures help to find and to quantify (the impacts of) causes of service variability (as shown by Fig. 2). This tool prevents expensive and lengthy field observations; it shows the main locations and times to visit for further analysis.

Concerning the causes, Fig. 7 demonstrates, for instance, too much slack in the timetable between stops 14 and 18: all trips (including the early ones) seem to win time with regard to the schedule. It is also shown that the holding regime effectively prevents vehicles to depart ahead of schedule.

Figure 8 shows the distribution of speed between stops. Due to interaction with much other public transport and limited traffic light priority, the differences between the 85 th and 15 th value are substantial at some links (e.g. stops 7-9).

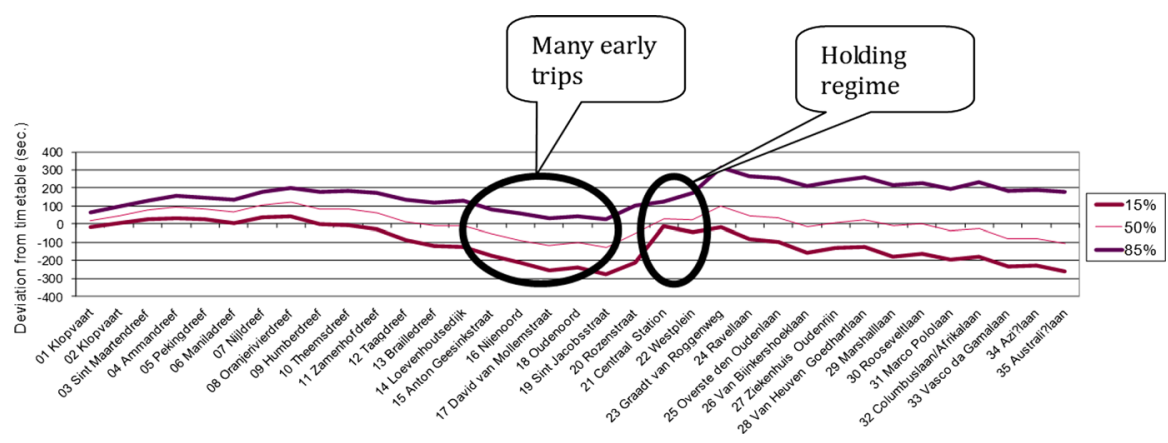

Fig. 7 GOVI-tool graph of schedule deviation, bus line 7 Utrecht, evening rush hour 


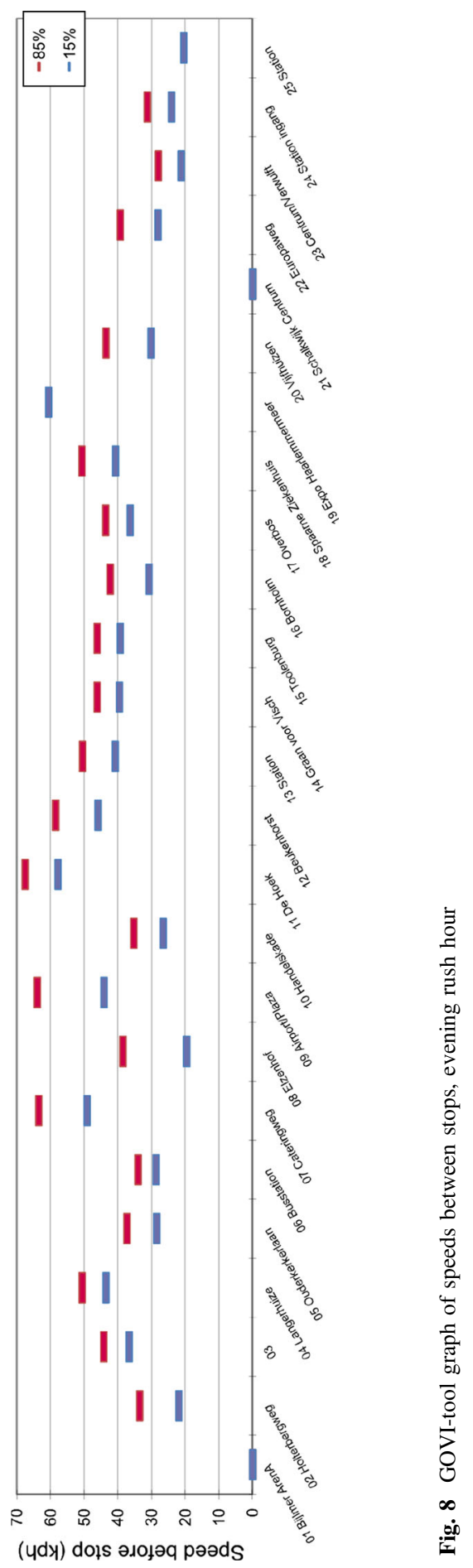




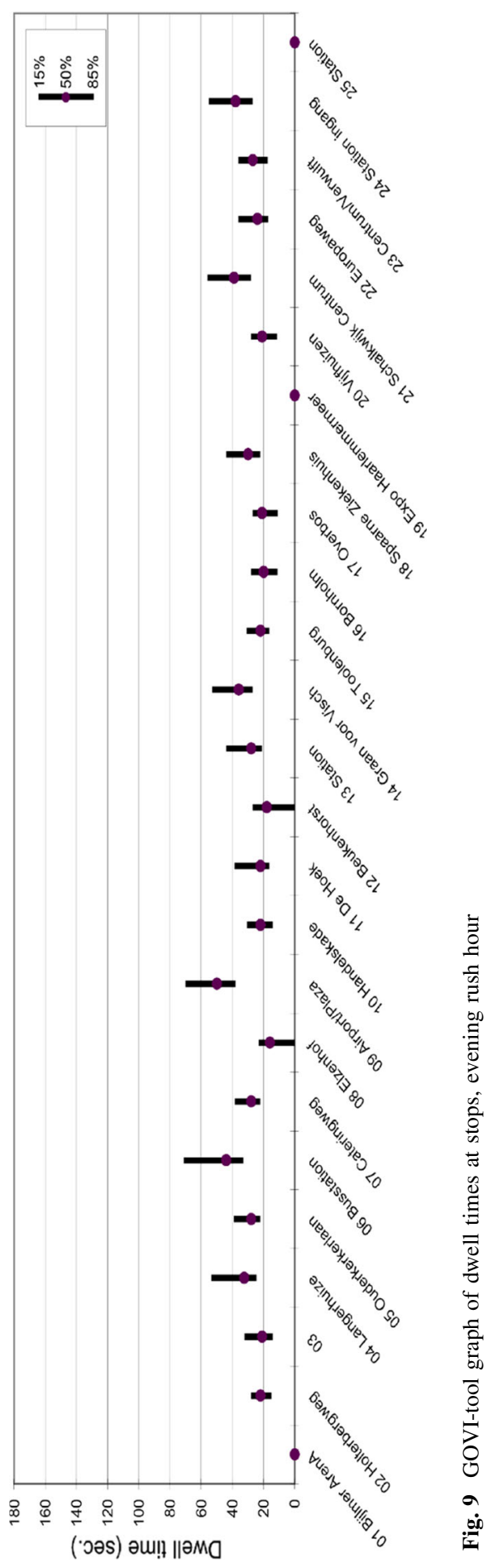


Figure 9 revealed the impact of driver relief at stop 6, resulting in a large distribution. Stops 8 and 12 face limited demand, which also results into a large distribution.

Figure 10 shows a list of bottlenecks found by the tool. For the case of Utrecht, Table 2 shows the relation between the threshold value for the average dwell times and the number of bottlenecks (i.e. stops where the average dwell time is larger than the indicated threshold value) found by the tool. There is a clear relation: the number of selected bottlenecks almost linearly decreases with the threshold value. Geographically speaking, the large dwell times of bus lines typically occur at the train station of Utrecht, where vehicles on through lines have to wait for their scheduled departure times.

The added value of these analyses using the GOVI-tool was that bottlenecks (with regard to travel time and service reliability) were identified quickly. In addition, the tool enabled the quantification of the (societal) costs of low quality of service and also the calculation of the expected benefits of improvements (such as synchronisation, exclusive infrastructure, transformation of a bus line into a tram line; see, e.g., Lee et al. 2014; Van Oort 2012). In these cases, passenger countings were added. Other applications will be presented in the following section.

\section{Discussion: vehicle vs. passenger}

Until now, commonly used indicators (like punctuality, as shown in the previous sections and figures) only focus on the vehicle, whereas a focus on the passenger is desired (for instance to support decisions). Therefore, we developed a new indicator: the average additional travel time due to unreliability (Van Oort and van Nes 2009). This indicator translates supply data (like punctuality) into passenger impacts; expressed in the additional time it takes a passenger to travel from origin to

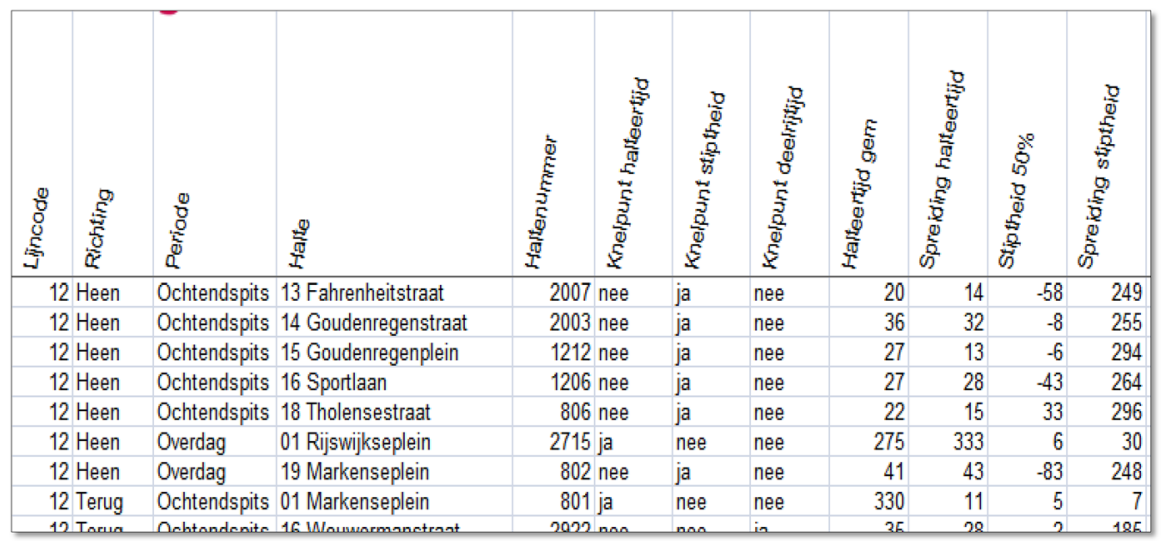

Fig. 10 GOVI-tool list of automatically found bottlenecks. Columns left to right: line, direction, period, stop, stop number, bottleneck yes/no: [dwell time, punctuality, trip time], avg. dwell time, distribution dwell time, punctuality median, distribution punctuality 
Table 2 Relation between threshold for average dwell time and number of selected bottlenecks for the Utrecht case
Threshold average dwell time (s) Number of selected bottlenecks

$\begin{array}{ll}60 & 678 \\ 120 & 556 \\ 180 & 450 \\ 240 & 314 \\ 300 & 191 \\ 360 & 112\end{array}$

destination due to unreliable operations. This time can be calculated for an entire service line or per stop. This enables taking unreliability into account when assessing policy measures, for example in social cost-benefit analysis. In combination with the travel time variation, these indicators cover the main effects of unreliability on travel time.

These indicators, additional travel time and travel time variation, can be calculated from operational data of vehicles and passengers (e.g. smartcard data): the combination of vehicle 'behaviour' and passenger behaviour determine the reliability effects (Van Oort and van Nes 2009). When additional travel time and travel time variation are known, the value of time and value of reliability can be used to monetize the societal effects (Significance 2012).

Due to the increasing availability of mentioned data (see, e.g., Van Oort et al. $2015 \mathrm{~b}$ for more details on the Dutch smartcard in addition to other international examples) and new insights into calculating passenger impacts, assessing passenger impacts in societal cost-benefit analyses becomes much easier and thus is more often applied. Several Dutch examples already included this indicator, as shown by Van Oort (2012).

Providing insights into the unreliability of transit lines is only the first step towards a better understanding of the effect of unreliability on passengers, since within one journey several transit lines may be used. The reliability of a passenger journey is determined by its weakest link: a reliable train ride is worth nothing if the connecting bus service is very unreliable, since the entire journey is then deemed unreliable. Consequently, a passenger may consider an alternative mode for the whole trip illustrating that train ridership is negatively influenced by the quality of the connecting bus service.

Insights into these network effects are only possible if all properties of various modes are taken into account. A transportation model can cope with these network effects. When reliability of public transport is integrated into transportation modelling, the (negative) influence of unreliability becomes clear. Furthermore, it becomes possible to investigate effects of policy measures that positively influence reliability on public transport ridership. In a transportation model, these effects are shown for the entire network, so that investments in a particular line may have influence on other lines as well. When costs and benefits are determined in this way, the results of social cost-benefit analyses will come closer to reality. The remaining question is how to take reliability into account in such models. First steps have been taken to operationalize this idea in the Dutch planning practice, by incorporating 
public transport reliability in the transportation model of the city region of Utrecht (Van Oort et al. 2015a). Connecting GOVI-data, processed by the GOVI-tool, to the transport model was the most essential step in this approach.

\section{Conclusions}

Since the funding of public transport is under growing pressure, the need to enhance the quality of services is increasing. The key elements for better and more efficient public transport are shorter and more reliable travel times. Removing bottlenecks in the operations implies that costs can be reduced while the quality of the service improves. This results in increasing ridership and revenues. This way, the cost effectiveness of public transport is improved in two parallel ways. This paper presented research on how available data sources may support these improvements in the Netherlands. We also showed the potential of the data with regard to individual driver assistance.

To find the bottlenecks and potential benefits of improvements, data of historical operations is very helpful. In the Netherlands, this data has recently become available via GOVI. The objective of the GOVI-system was to facilitate the provision of dynamic travel information, but the recorded data also provide huge insights into actual and scheduled (historic) performance. We developed a tool to translate all these data into easily accessible information so that bottlenecks can be identified and measures can be taken by the transport authorities and operators to solve them.

A next step in the development of the tool is translating vehicle data into passenger impact. Since additional data on passenger behaviour and flows become available (via smartcards for instance), service reliability impacts per passenger per stop may be calculated (i.e., additional travel time and its distribution; Van Oort et al. 2015a).

The following step is to use this knowledge on a network level. The city region of Utrecht started this development by incorporating public transport reliability into its regional multimodal transportation model. In this way, the model is better equipped to determine network effects of policy measures that influence transit reliability. Societal costs and benefits of measures aiming at improved service reliability may be explicitly accounted for, thereby supporting decision-making. GOVI-data proved to be a very valuable source for this approach.

Acknowledgments This research is performed in cooperation with BRU, the transit authority in the region Utrecht, the Netherlands; Delft University of Technology, Department of Transport and Planning; Goudappel Coffeng mobility consultants; the Dutch Organisation for Scientific Research (NWO); and the Dutch OpenGeo Foundation. The authors thank their partners for their support.

Open Access This article is distributed under the terms of the Creative Commons Attribution 4.0 International License (http://creativecommons.org/licenses/by/4.0/), which permits unrestricted use, distribution, and reproduction in any medium, provided you give appropriate credit to the original author(s) and the source, provide a link to the Creative Commons license, and indicate if changes were made. 


\section{References}

Abkowitz M, Engelstein I (1983) Factors affecting running time on transit routes. Transp Res Part A 17(2):107-113

Abkowitz M, Tozzi J (1987) Research contributing to managing transit service reliability. J Adv Transp 21:47-65

Balcombe R, Mackett R, Paulley N, Preston J, Shires J, Titheridge H, Wardman M, White P (2004) The demand for public transport: a practical guide

Bates J, Polak J, Jones P, Cook A (2001) The valuation of reliability for personal travel. Transp Res Part E 37:191-229

Bregman S (2012) Uses of social media in public transportation. Transit Cooperative Research Program (TCRP) Synthesis 99. Transportation Research Board, Washington

Calabrese F (2011) Estimating origin-destination flows using mobile phone location data. In: Pervasive computing, IEEE, pp 36-44

Cats O (2014) Regularity-driven bus operations: principles, implementation and business models. Transp Policy 36:223-230

Ceder A (2007) Public transit planning and operation, theory, modelling and practice. Technion-Israel Institute of Technology, Haifa

Delgado F, Munoz JC, Giesen R (2012) How much can holding and/or limiting boarding improve transit performance? Transp Res Part B Methodol 46(9):1202-1217

Fernandez R, Tyler N (2010) Influence of platform height, door with and fare collection on bus dwell time, Laboratory evidence for Santiago de Chile. In: Proceedings of 89th annual meeting of Transportation Research Board, Washington, DC

Furth PG, Muller THJ (2009) Optimality conditions for public transport schedules with timepoint holding. Public Transp 1(2):87-102

Furth PG, Hemily B, Muller THJ, Strathman JG (2006) TCRP Report 113: using archived AVL-APC data to Improve transit performance and management. Washington, DC

Goverde RMP, Hooghiemstra G, Lopuhaä HP (2001) Statistical analysis of train traffic: the Eindhoven case, TRAIL Studies in Transportation Science, No. S2001/1, DUP Science, Delft

Goverde RMP, Meng L (2011) Advanced monitoring and management information of railway operations. J Rail Transp Plan Manag 1(2):69-79

Hickman M (2004) Evaluating the benefits of bus automatic vehicle location (AVL) systems. In: Levinson D, Gillen D (eds) Assessing the benefits and costs of intelligent transportation systems. Kluwer, Boston

Hofmann M, Mahony MO (2005) The impact of adverse weather conditions on urban bus performance measures. In: Proceedings of the 8th international IEEE conference on intelligent transportation systems, Vienna, pp 84-89

http://9292opendata.org/ (Accessed 18 July 2013)

http://bison.connekt.nl/ (Accessed 18 July 2013)

http://govi.nu (Accessed 18 July 2013)

http://webwereld.nl/cloud/56325-gvb-maakt-real-time-reisinformatie-open-data (Accessed 18 July 2013)

http://www.openov.nl/ (Accessed 18 July 2013)

Kanacilo EM, van Oort N (2008) Using a rail simulation library to assess impacts of transit network planning on operational quality. In: Allen J, Arias E, Brebbia CA, Goodman CJ, Rumsey AF, Sciutto G, Tomii N (eds) Computers in railways XI. WIT Press, Southampton, pp 35-44

König A, Axhausen KW (2002) The reliability of the transportation system and its influence on the choice behaviour. In: Proceedings of the 2nd Swiss transportation research conference, Monte Verità

Landex A, Kaas AH (2009) Examination of operation quality for high-frequent railway operation. In: Proceedings of 3rd international seminar on railway operations modelling and analysis, Zürich

Lee A, van Oort N, van Nes R (2014) Service reliability in a network context. Transp Res Rec 2417:18-26

Levinson HS (1983) Analyzing transit travel time performance. Transp Res Rec 915:1-6

Li Z, Hensher DA, Rose JM (2010) Willingness to pay for travel time reliability in passenger transport: a review and some new empirical evidence. Transp Res Part E 46:384-403

Mazloumi E, Currie G, Rose G (2010) Using GPS data to gain insight into public transport travel time variability. J Transp Eng 136(7):623-631 
Neema N, Hickman M, Ma Z-L (2015) Activity detection and transfer identification for public transit fare card data. Transportation 42(4):683-705

Noland R, Small KA (1995) Travel time uncertainty, departure time and the cost of the morning commute. In: Proceedings of the 74th annual meeting of the Transportation Research Board, Washington, DC

OECD/International transport forum (2010) Improving reliability on surface networks. OECD Publishing, Paris

Pelletier M, Trepanier M, Morency C (2011) Smart card data use in public transit: a literature review. Transp Res Part C Emerg Technol 19(4):557-568

Schmöcker JD, Bell MGH (2002) The PFE as a tool for robust multi-modal network planning. Traffic Eng Control 44(3):108-114

Shalaby A, Lyon C, Sayed T (2001) Transferability of travel time models and provision of real-time arrival time information. In: Proceedings of intelligent transportation systems conference, Oakland, pp 982-987

Significance, VU University Amsterdam, and John Bates Services (2012) Values of time and reliability in passenger and freight transport in the Netherlands: report for the Ministry of Infrastructure and the Environment

Snelder M, Tavasszy LA (2010) Quantifying the full reliability benefits of road network improvements. In: Proceedings of WCTR 2010, Lisbon

Sparing D, Goverde RMP (2013) Identifying effective guaranteed connections in a multimodal public transport network. Public Transp 5(1-2):79-94

Strathman JG, Kimple T, Dueker K, Gerhart R, Callas S (2000) Service reliability impacts of computeraided dispatching and automatic location technology: a Tri-Met case study. Transp Q 54(3):85-102

Turnquist MA, Bowman LA (1980) The effects of network structure on reliability of transit service. Transp Res Part B 14:79-86

van den Heuvel J, Voskamp A, Daamen W, Hoogendoorn SP (2015) Using Bluetooth to estimate the impact of congestion on pedestrian route choice at train stations. In: Charaibi M (ed) Proceedings of traffic and granular flow '13, Switzerland, pp 73-82

Van Der Waard J (1988) The relative importance of public transport trip time attributes in route choice. In: Proceedings PTRC, London, pp 67-78

Van Oort N (2011) Service reliability and urban public transport design, T2011/2, TRAIL PhD Thesis Series, Delft

Van Oort N (2012) Quantifying benefits of enhanced service reliability in public transport. In: Munoz JC (ed) Proceedings of the 12th international conference on advanced systems for public transport (CASPT12)

Van Oort N (2014) Incorporating service reliability in public transport design and performance requirements: international survey results and recommendations. Res Transp Econ 48:92-100

Van Oort N, van Nes R (2009) Line length versus operational reliability: network design dilemma in urban public transportation. Transp Res Rec 2112:104-110

Van Oort N, van Nes R (2010) The impact of rail terminal design on transit service reliability. Transp Res Rec 2146:109-118

Van Oort N, Boterman JW, van Nes R (2012) The impact of scheduling on service reliability: trip-time determination and holding points in long-headway services. Public Transp 4(1):39-56

Van Oort N, Brands T, de Romph E, Flores JA (2015a) Unreliability effects in public transport modelling. Int J Transp 3(1):113-130

Van Oort N, Brands T, de Romph E (2015b) Short term ridership prediction in public transport by processing smart card data. Transportation Research Record (in press)

Vuchic VR (2005) Urban transit, operations, planning and economics. Wiley, New Jersey

Waterson B, Rajbhandary B, Hounsell N (2003) Simulating the impacts of strong bus priority measures. J Transp Eng 129:642-647

Weidmann U (1995) Grundlagen zur Berechnung der Fahrgastwechselzeit, Schriftenreihe des IVT No. 106, Institut für Verkehrsplanung und Transporttechnik, ETH Zürich (in German)

Wilson NHM, Zhao J, Rahbee A (2009) The potential impact of automated data collection systems on urban public transport planning. In: Schedule-based modeling of transportation networks. Springer, Berlin, pp 75-99

Xuan Y, Argote J, Daganzo CF (2011) Dynamic bus holding strategies for schedule reliability: optimal linear control and performance analysis. Transp Res Part B Methodol 45(10):1831-1845 